\title{
Study on Multi-Source Localization of Wireless Sensor Network Based on Quantitative Information
}

\author{
Yunting Liu, Hui Guo, Yuanwei Jing and Siying Zhang \\ School of Information Science and Engineering, Northeastern University, \\ Shenyang 110819, China \\ School mechanical engineering, Shenyang University of Technology, Shenyang \\ 110870, China \\ liuyunting0224@163.com
}

\begin{abstract}
Against the problem that quantitative localization is concentrated on single-source localization, this paper studies the quantitative multi-source localization methods based on wireless sensor network. Firstly, the log-quantization strategy which reflects the characteristics of acoustic source is proposed. The sensor node transmits the quantized information to base station according to the measurements and log-quantization strategy. Secondly the based station estimates the location of the sources according to the proposed method termed as multiple source localization based on possibilistic $C$ means clustering algorithm. Finally the efficient of the proposed method is verified by the simulation under different parameters. The simulation results show that the proposed method could accurately estimate the location of source. And the proposed method is robust to the packet loss rate.
\end{abstract}

Keywords: Wireless Sensor Networks, Localization, Quantized Information, Acoustic Source, Possibilistic C Means Clustering

\section{Introduction}

With the constant development of micro-electronics, short-distance communication and sensing technology, wireless sensor networks (WSNs) have become a research hotspot ${ }^{[1]}$. Signal source (or target) localization is an important research direction of WSNs, and the results of the researches can be widely applied into the areas of the localization of vehicles, large underwater creatures and humans. WSNs-based signal source localization can be categorized into three types according to the methods of measurement, namely TDOA (Time Difference of Arrival)-based, RSS (Received Signal Strength)-based and AOA (Angle of Arrival)-based localization. Among them, TDOA-based method places a relatively high demand on the time synchronization of nodes and AOA-based method places a relatively high demand on node hardware for the need of array antenna. However, RSS-based method places a relatively low demand on node hardware and the power dissipation is relatively small, so it best suits the application of WSNs ${ }^{[2]}$.

WSNs-based multi-source localization has attracted the attention of scholars from both China and beyond. Sheng [3] put forward the maximum likelihood estimation (MLE) to establish the objective function for multi-source localization, and then the localization of signal sources is searched and estimated through adopting the expectation maximization and multi-resolution. Ampeliods [4] propose to adopt the alternate mapping algorithm to divide the localization issue of multi-sources into several non-convex optimization problems, which significantly lowers the computing complexity when compared with MLE-based method. Shen [5] break the complex multi-source localization problem into 
several convex optimization ones, based on which TTS (Tractable Three-Step) algorithm is put forward to estimate the location of signal sources.

The above algorithms require the sensor nodes to transmit the measured original data to base stations, which then estimate the localization of signal sources according to measurements and sensor nodes. However, the energy of sensor nodes and the communication bandwidth are extremely limited and the transmission of original data consumes relatively much energy, so the measured original data can be quantized so that the nodes only need to transmit several bits of information after quantization, hence significantly lowering the transmission quantity of data and lowering the energy consumption of nodes. Ozdemir [6] propose a target localization method based on quantitative data, which establishes the objective function through MLE-based method, obtains the estimated localization of targets through optimizing the objective function, and integrates the channel uncertainty into it, so this method has certain fault tolerance for the channel disturbance. Masazade [7] put forward cyclic source localization algorithm against heterogeneous sensor networks, which firstly obtains the posterior probability density function through the Monte-Carlo method, then proposes two node selection methods, and finally estimates the location of signal sources according to the selected nodes and objective function. Vempaty [8] put forward a cyclic localization method based on coding theory. In each cycle, the base stations estimate the location of signal sources though solving the problem of M-nary hypothesis testing and meanwhile determine the areas of interest of the next cycle. Compared with the maximum likelihood estimation, this method lowers the computing complexity.

At present, researches on quantitative localization are mostly concentrated on singletarget (single-source) localization, so there are few literatures relevant to the multi-source localization methods based on quantitative information. Based on the above analyses, this paper studies the multi-source localization methods based on quantitative information, and puts forward a multi-source localization method based on modified possibilistic Cmeans Clustering Algorithm.

\section{System Mobel}

Suppose that $N$ sensor nodes are randomly deployed in the sensing field, the location of each sensor node is known, there are $K$ acoustic sources in the field with each one of them sending acoustic signals evenly to all around, and the influence of obstacles on signal propagation is not considered, then the signal intensity received at the $i$-th sensor node is [3]:

$$
y_{i}=\sum_{k=1}^{K} \frac{S_{k}}{d_{i k}^{\gamma / 2}}+n_{i}
$$

Where, $y_{i}$ is the received energy of acoustic signal at $i$-th sensor node, $n_{i}$ is the measurement noise and it is modeled as zero mean Gaussian noise with variance $\sigma^{2}$; $S_{k}$ is the energy of $k$-th signal source, and $\gamma$ is the path loss coefficient, $d_{i k}=\left\|\rho_{k}-r_{i}\right\|$ is the Euclidean distance between the $i$-th node and the $k$-th signal source, $\rho_{k}$ is the coordinate of the $k$-th signal source, and $r_{i}$ is the coordinate of the $i$-th sensor node.

Measurement $y_{i}$ at $i$-th sensor node is quantized into $M$ bits, shown as $D_{i}$. Assume $L=2^{M}$, and $D_{i}$ is an integer within the range of $[0, L-1]$. The quantization of $i$-th sensor node can be shown as: 


$$
D_{i}=\left\{\begin{array}{cc}
0, & y_{i} \leq \eta_{1} \\
1, & \eta_{1}<y_{i} \leq \eta_{2} \\
\vdots & \vdots \\
L-1, & y_{i}>\eta_{L-1}
\end{array}\right.
$$

Where, $\eta_{i}$ is the quantization threshold.

As shown in Fig. 1, in the situation of one signal source, measurement $y_{i}$ decreases sharply with the increase of distance. The adoption of traditional uniform quantization will lower the resolution when the measurement is relatively small. Therefore, this paper proposes the logarithmic quantization strategy. Aiming at a certain target source measured by the sensor network, its energy value will often fall into the range of $S_{M I N} \leq S_{k} \leq S_{M A X}$, then the quantization threshold can be defined as follows:

$$
\eta_{i}=10^{\left[a_{1}+\frac{(i-1)\left(a_{2}-a_{1}\right)}{L-2}\right]}, i=1, \ldots, L-1
$$

Where, $a_{1}=\log _{10}\left(K \cdot S_{\min }\right), a_{2}=\log _{10}\left(K \cdot S_{\max }\right)$.

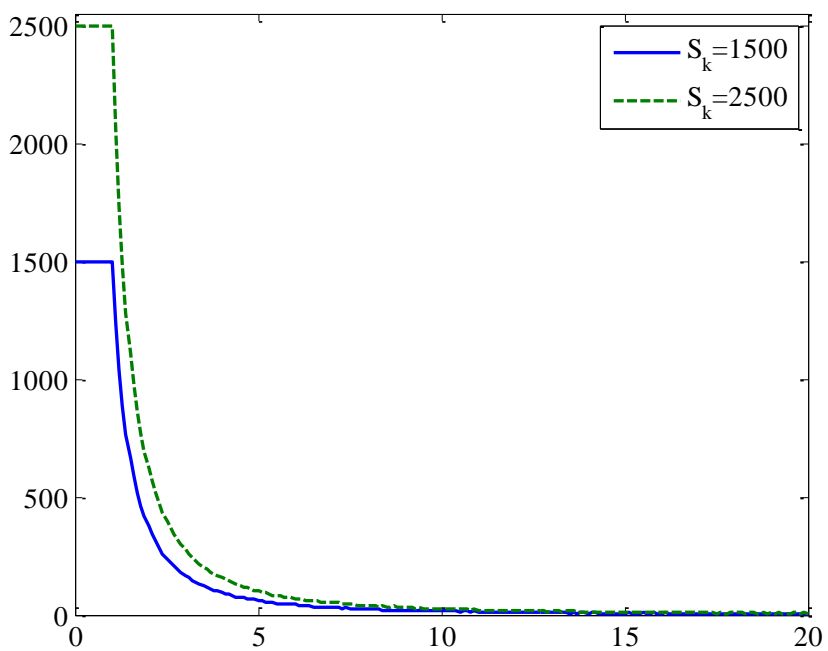

Figure 1. The Relationship between Measurement and Distance

The maximum likelihood estimation is a multi-source localization algorithm that has been widely applied, but its computing complexity is high, so it is unsuitable for the sensor nodes with limited calculation and storage capacity.

\section{Multi-Source Localization Algorithm Based on Modified Possibilistic C-Means}

\subsection{Possibilistic C-Means Clustering Algorithm}

In 1993, R. Krishnapuram and J. Keller introduced possibility theory into clustering and put forward Possibilistic C-Means Clustering Algorithm (PCMC) ${ }^{[9]}$. Compared with fuzzy C-means, it lifts the restrictions on sample membership degree, or in other words, the sum of membership degree will not be limited to 1 .

Given the data set that has been classified into $\mathrm{C}$ types, through minimizing the following function we can obtain as ${ }^{[10]}$ : 


$$
J(t)=\sum_{i=1}^{C} \sum_{j=1}^{q}\left(t_{i j}\right)^{m}\left\|x_{j}-p_{i}\right\|^{2}+\sum_{i=1}^{C} \lambda_{i} \sum_{j=1}^{q}\left(1-t_{i j}\right)^{m}
$$

Where $t_{i j} \in[0,1], 0<\sum_{j=1}^{q} t_{i j}<q, p_{i}$ is the $i$-th clustering center, $C$ is the number of clustering, $t_{i j}$ is the probable subdivision value of $\mathrm{xj}$ to $\mathrm{i}$ type, $\mathrm{m}$ is the weighted index ( $m=2$ in this paper), and is the penalty factor.

The expression of penalty factor is as follows:

$$
\lambda_{i}=W \frac{\sum_{i=1}^{q} t_{i j}^{m}\left\|x_{j}-p_{i}\right\|^{2}}{\sum_{i=1}^{q} t_{i j}^{m}}
$$

Where $W=1$.

Through iteration, we can obtain the probable subdivision value and clustering centers as:

$$
\begin{gathered}
t_{i j}=\left[1+\left[\frac{\left\|x_{j}-p_{i}\right\|^{2}}{\lambda_{i}}\right]^{\frac{1}{m-1}}\right]^{-1} \\
p_{i}=\frac{\sum_{j=1}^{q} t_{i j}^{m} x_{j}}{\sum_{j=1}^{q} t_{i j}^{m}}
\end{gathered}
$$

\subsection{Multi-Source Localization Algorithm}

Assume that nodes with $D_{i}>0$ are the alarm nodes, each alarm node directly transmit the quantized data $D_{i}$ to base stations, and base stations will estimate the location of signal sources according to the proposed multi-source localization algorithm. $R=\left[r_{1}, r_{2}, \ldots, r_{q}\right]$ is the coordinate set of alarm nodes, and $q \leq N$. Adopting the following modified possibilistic C-means algorithm can estimate the location of signal sources.

$D_{i}$ is normalized:

$$
D_{i}^{\prime}=\frac{D_{i}}{\sum_{i=1}^{n} D_{i}}
$$

Based on (1) and (2), the larger the quantized value $D_{i}$, the larger the measurement of sensor nodes, which indicates that if the sensor node is closer to signal source, then its weight in calculating clustering center will be bigger. Therefore, this paper takes $D_{i}^{\prime}$ as the weight to redefine the clustering center as follows: 


$$
p_{i}=\frac{\sum_{j=1}^{n}\left(t_{i j}\right)^{m} D_{j}^{\prime} x_{j}}{\sum_{j=1}^{n}\left(t_{i j}\right)^{m} D_{j}^{\prime}}
$$

The steps of the proposed algorithm are as follows:

Step 1. Initialize parameters: the number of clustering $C=K$, the number of initial iteration $t=1$, the biggest number of iteration is $t_{\max }$, and the stopping threshold $\varepsilon=0.01$. Initialization probable subdivision value is $t_{i j}^{(0)}$ and the clustering center is $p_{i}^{(0)}$. Based on (5), the penalty factor $\lambda_{i}$ can be calculated.

Step 2. Calculate probable subdivision values according to (6).

Step 3. Calculate clustering center according to (9).

Step 4. Calculate objective function value according to (4), and $t=t+1$.

Step 5. If $\|J(t)-J(t-1)\|<\varepsilon$ or $t>t_{\max }$, then stop; or if otherwise, repeat step 2-4.

Clustering center $P=\left[p_{1}, \ldots, p_{K}\right]$ will be regarded as the estimated location of the signal sources.

\section{Simulation Results}

The simulation environment in this paper is as follows: in a field with an area of $100 \mathrm{~m} \times 100 \mathrm{~m}$, randomly deploy $N$ sensor nodes and $K$ signal sources, and all default parameters are shown in Table 1.

Table 1. The Default Parameters

\begin{tabular}{c|c|c}
\hline Parameter & Symbol & Default \\
\hline Node number & $N$ & 400 \\
Signal source number & $K$ & 2 \\
Measurement noise SD & $\sigma$ & $1 \mathrm{~m}$ \\
Quantized bit number & $M$ & $3 \mathrm{bit}$ \\
Energy at $k$ signal source & $S_{k}$ & 1500 \\
\hline
\end{tabular}

The simulation results of this paper are obtained through running the Monte Carlo experiments for 2000 times, and following indexes are adopted in assessing the localization errors:

$$
\frac{1}{R \cdot K} \sum_{i=1}^{R} \sum_{k=1}^{K} \sqrt{\left(x_{k}-\hat{x}_{k}(i)\right)^{2}+\left(y_{k}-\hat{y}_{k}(i)\right)^{2}}
$$

Where $\left(x_{k}, y_{k}\right)$ is the real location of the $k$-th signal source, $\left(\hat{x}_{k}(i), \hat{y}_{k}(i)\right)$ is the estimated location of the $k$-th signal source after running Monte Carlo experiment for the $i$-th time, and $R=2000$.

Fig. 2 presents the relationship between the noise standard deviation $\sigma$ and the localization error under different quantization bit numbers. As can be seen from the Fig. 2, the localization error of the proposed algorithm increases with the increase of $\sigma$. The reason lies in the fact that a larger $\sigma$ will cause the bigger the disturbance 
of noise to measurement, and the larger probability for errors of quantized value $D_{i}$. With increasing number of quantization bit $M$, the localization error gradually decreases. The reason lies in the fact the bigger $M$ is, the more the quantitative information supplied by nodes to base stations will be, thus uplifting the accuracy of localization.

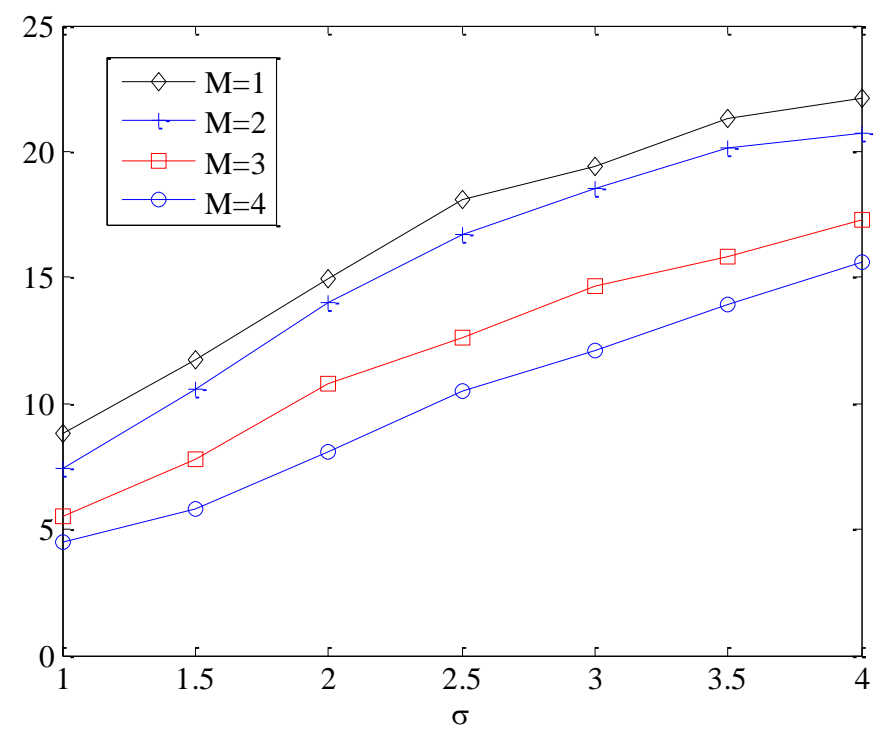

\section{Figure 2. The Relationship between Localization Error and Standard Variance of Noise under Different Quantization Bit Numbers}

Table 2. Presents the Influence of Node Number and Signal Source Energy. $S_{k}$ on localization error. It can be seen that the accuracy of the proposed algorithm increases with the increase of node number, because the more the number of node, the more effective information is supplied, hence improving the accuracy of localization. Due to the fact the disturbance among signals will increase as the signal energy $S_{k}$ becomes bigger, the accuracy of localization is negatively influenced.

Table 2. The Localization Error

\begin{tabular}{cccccc}
\hline & $N=200$ & $N=250$ & $N=300$ & $N=350$ & $N=400$ \\
\hline$S_{K}=1500$ & 6.0581 & 5.8402 & 5.756 & 5.624 & 5.624 \\
$S_{K}=2000$ & 7.3731 & 7.2468 & 7.1978 & 7.1559 & 7.0534 \\
$S_{K}=2500$ & 8.763 & 8.6856 & 8.5772 & 8.5056 & 8.38 \\
\hline
\end{tabular}

The above two conclusions are from relatively ideal situations, or in other words, sensor nodes can all transmit quantized value $D_{i}$ to base stations. In real situations, due to reasons like node malfunction or channel congestion, packet loss might arise, or in other words, base stations fail to receive the quantized values of some nodes. Fig. 3 presents the relationship between packet loss rate and localization error. It can be seen that with increasing packet loss rate, the localization error of the proposed algorithm does not vary basically. Therefore, the proposed algorithm has 
certain robustness. Meanwhile, with increasing noise standard deviation $\sigma$, the localization error climbs up.

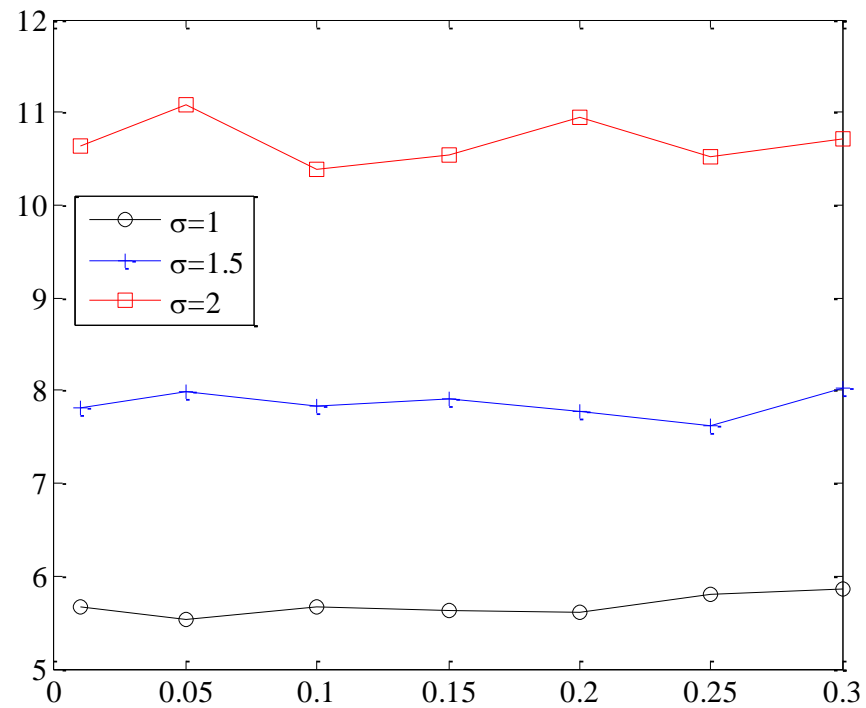

Figure 3. The Relationship between Packet Loss Rate and Localization Error

Assume that the node communication radius is $75 \mathrm{~m}$, and each node transmits its own 16 bits of ID information (network address) and measured energy information to base stations in a single-hop manner. If the energy of the $k$-th signal source $S_{k}=1500$, then the data packet required to be transmitted according to traditional methods based on measurement is 27 bits in size (16bit+11bit). If the method based on quantization proposed in this paper, then only 19 bits of data need to be transmitted (16bit +3 bit, here assume the quantization bit number $M=3$ ).

Given that the measurement data is sent in a single-hop manner with no need to transmit data from other nodes, so the experimental model adopted in this experiment is as follows ${ }^{[11]}$ :

$$
E_{t x}(l, d)=l \times E_{\text {ele }}+l \times d^{2} \times E_{\text {amp }}
$$

Where, $E_{\text {ele }}$ is the energy consumed by non-transmission equipment (frequency synthesizer, mixer and filter), $E_{a m p}$ is the energy consumed by projectors, $l$ is the size of data packet, and $d$ is the transmission distance.

From the above, it can be seen that under the condition that the transmission distance is fixed, the node energy consumption is in direct proportion to the size of data packet. Default parameters adopted in this experiment are as shown in the following Table 3 .

Table 3. Default Parameters of the Energy Simulation

\begin{tabular}{cc}
\hline Parameter & Value \\
\hline$E_{\text {ele }}$ & $3.63 \mu \mathrm{J} / \mathrm{bit}$ \\
$E_{\text {amp }}$ & $16.3 \mu \mathrm{J} / \mathrm{bit}$ \\
$d$ & $75 \mathrm{~m}$ \\
\hline
\end{tabular}




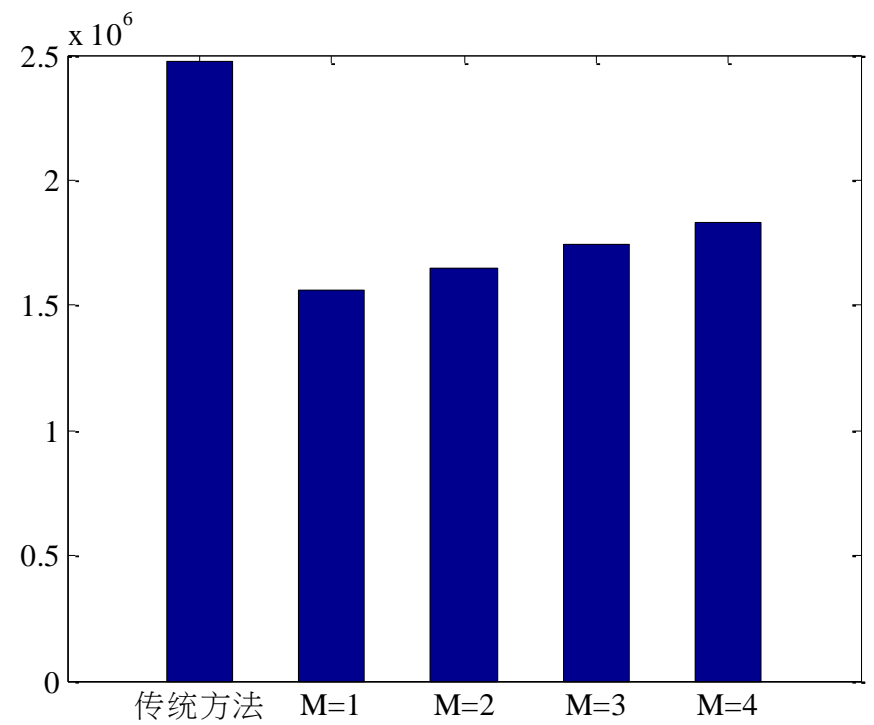

Figure 4. Comparison of Energy Consumption

\section{Conclusions}

This paper studies the multi-source localization methods of quantitative information based on wireless sensor network. Firstly, aiming at the propagation features of acoustic sources, it proposes logarithmic quantization strategy, which can better reflect the relationship between acoustic source and distance. Then, a multi-source localization method based on possibilistic C-means clustering (PCMC) is proposed to transform multi-source localization problem into clustering problem, with a view to obtaining the estimated location of signal sources through calculating the clustering center. The simulation results show that the algorithm in this paper can accurately estimate the location of multiple signal sources, with certain robustness against packet loss probability. Compared with traditional localization methods based on measurement, the algorithm proposed in this paper can effectively reduce energy consumption.

\section{Acknowledgements}

In this paper, the research was sponsored by the Special Scientific Research Fund of Forestry Public Welfare Industry (201304515-3), National Natural Science Fund Program (63104021), Special Fund Program for Basic Research Business Expenses of Central Universities (N110404032)

\section{References}

[1] J. Wang, Q. Gao, P. Cheng, Y. Yu, K. Xin and H. Wang, "Lightweight Robust Device-Free Localization in Wireless Networks", IEEE Transactions on Industrial Electronics, vol.6, no. 10, (2014), pp. 5681-5689.

[2] H. Lohrasbipeydeh, T. A. Gulliver and H. Amindavar, "Blind Received Signal Strength Difference Based Source Localization with System Parameter Errors", IEEE Transactions on Signal Processing, vol. 62, no. 17, (2014), pp. 4516-4531.

[3] X. H. Sheng and Y. H. Hu, "Maximum likelihood multiple-source localization using acoustic energy measurements with wireless sensor networks", IEEE Transactions on Signal Processing, vol. 53, no. 1, (2005), pp. 44-53.

[4] D. Ampeliods and K. Berberidis, "Low complexity multiple acoustic source localization in sensor networks based on energy measurements", Signal Processing, vol. 90, no. 4, (2010), pp. 1300-1312. 
[5] H. Shen, Z. Ding and S. Dasgupta, "Multiple Source Localization in Wireless Sensor Networks Based on Time of Arrival Measurement", IEEE Transactions on Signal Processing, vol. 62, no. 8, (2014), pp. 1938-1949.

[6] Ozdemir, R. Niu and P. K. Varshney, "Channel Aware Target Localization with Quantized Data in Wireless Sensor Networks", IEEE Transactions on Signal Processing, vol. 57, no. 3, (2009), pp. 1190-1202.

[7] E. Masazade, R. Niu and P.K. Varshney, "Energy Aware Iterative Source Localization for Wireless Sensor Networks", IEEE Transactions on Signal Processing, vol. 58, no.9 ,(2010), pp. 4824-4835.

[8] Vempaty, Y.S. Han and P. K. Varshney, "Target Localization in Wireless Sensor Networks Using Error Correcting Codes", IEEE Transactions on Information Theory, vol. 60, no. 1, (2014), pp. 697-712.

[9] C. Chuang, J.T. Jeng and S.C. Chang, "Hausdorff Distance Measure based Interval Fuzzy Possibilistic C-Means Clustering Algorithm”, International Journal of Fuzzy Systems, vol. 15, no. 4, (2013), pp. 471-479.

[10] S. Bahrampour, B. Moshiri and K. Salahshoor, "Weighted and Constrained Possibilistic Cmeans Clustering for Online Fault Detection and Isolation", Applied Intelligence, vol. 35, no. 2, (2011), pp. 269-284.

[11] M. Tariq, M. Macuha, Y. J. Park and T. Sato, "A Realistic Communication Model for Distributed Error-Prone Wireless Sensor Networks", IEICE Transactions on Communications. vol. 94, no.10, (2011), pp. 2805-2816.

[12] J. Hu, Z. Gao and W. Pan, "Multiangle Social Network Recommendation Algorithms and Similarity Network Evaluation [J]". Journal of Applied Mathematics, 2013 (2013).

[13] Z. Lv, T. Yin, Y. Han, Y. Chen and G. Chen, "WebVR-web virtual reality engine based on P2P network", Journal of Networks. vol. 6, no. 7, (2011), pp. 990-998.

[14] J. Yang, B. Chen, J. Zhou and Z. Lv, "A portable biomedical device for respiratory monitoring with a stable power source", Sensors, (2015).

[15] Z. Dongfang, "FusionFS: Toward supporting data-intensive scientific applications on extreme-scale high-performance computing systems", Big Data (Big Data), 2014 IEEE International Conference on. IEEE, (2014).

[16] S. Dang, J. Ju, D. Matthews, X. Feng and C. Zuo, "Efficient solar power heating system based on lenticular condensation. Information Science", Electronics and Electrical Engineering (ISEEE), 2014 International Conference on, (2014), pp. 26-28.

[17] X. Zhang, Y. Han, D. Hao and Z. Lv, “ARPPS: Augmented Reality Pipeline Prospect System", 22th International Conference on Neural Information Processing (ICONIP 2015), Istanbul, Turkey, In press.

[18] J. Hu and Z. Gao, "Distinction immune genes of hepatitis-induced heptatocellular carcinoma [J]", Bioinformatics, vol. 28, no. 24, (2012), pp. 3191-3194.

[19] Z. Su, X. Zhang and Z. Ou, "After we knew it: empirical study and modeling of costeffectiveness of exploiting prevalent known vulnerabilities across iaas cloud", Proceedings of the 9th ACM symposium on Information, computer and communications security, ACM, (2014).

\section{Author}

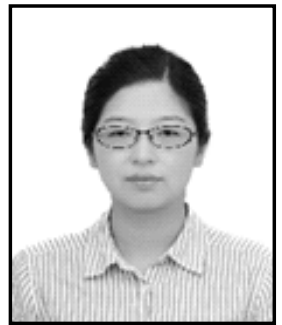

Yunting Liu was born in Shenyang, China, in 1983. She is currently studying for her Ph.D. degree in the major of Control Theory and Control Engineering at Northeastern University. She has received her M.S. degree in the major of Control Theory and Control Engineering at Northeastern University. Her main research interest lies in wireless sensor network. She has published several research papers in scholarly journals in the above research areas. 
International Journal of Future Generation Communication and Networking Online Vol. 9, No. 4, (2016) 